\title{
Multi-modal Medical Volumes Fusion by Surface Matching
}

\author{
Ayman M. Eldeib, Sameh M. Yamany, and Aly A. Farag \\ Computer Vision and Image Processing Laboratory \\ University of Louisville, Louisville, Kentucky 40292, USA \\ \{ayman, yamany, farag\}@cvip.uofl.edu
}

\begin{abstract}
This paper presents a fast, six degrees of freedom registration technique to accurately locate the position and orientation of medical volumes (obtained from $\mathrm{CT} / \mathrm{MRI}$ scans for the same patient) with respect to each other. The main contribution of this work is the use of a novel surface registration technique followed by a volume registration approach. The advantage of this combination is to have an accurate alignment and to reduce the time needed for registration. The surface registration uses a new surface representation scheme that captures the curvature information and codes it into 2-D images. Matching these images enables the recovery of the transformation parameters. For the multi-modal volume registration, the maximization of Mutual Information (MI) is used as a matching criterion and is enhanced by a new genetic based search technique. The results demonstrate that the new two-stage registration technique presented in this paper is robust in terms of speed and accuracy and allows for completely automatic registration of multimodality medical volumes.
\end{abstract}

\section{Introduction}

Medical imaging modalities produce different diagnostic information which complement each other. Skin and bone can be more easily extracted from Computed Tomography (CT) images than from Magnetic Resonance (MR) images. Also, in radiotherapy planning, CT data is used for dosage calculation, while soft tissues and tumor outlining is better segmented and extracted from the corresponding MR data. In many cases, other imaging modalities are needed for more diagnostic information, such as Magnetic Resonance Angiography (MRA) for extracting blood vessels, and Positron-Emission tomography (PET) for providing information on brain function. For our surgical planning system, navigation system and virtual neuroendoscope, we need all this information together. The objective of this work is to register data from multiple modalities into a single 3 -D model using a high fidelity fusion method. Figure 1 shows a block diagram of the proposed system. This paper is organized as follows. Section 2 introduces the surface registration technique. Maximization of mutual information using genetic algorithms is described in Section 3. Results are discussed in Section 4 and conclusions are given in Section 5 .

C. Taylor, A. Colchester (Eds.): MICCAI'99, LNCS 1679, pp. 672-679, 1999.

(C) Springer-Verlag Berlin Heidelberg 1999 


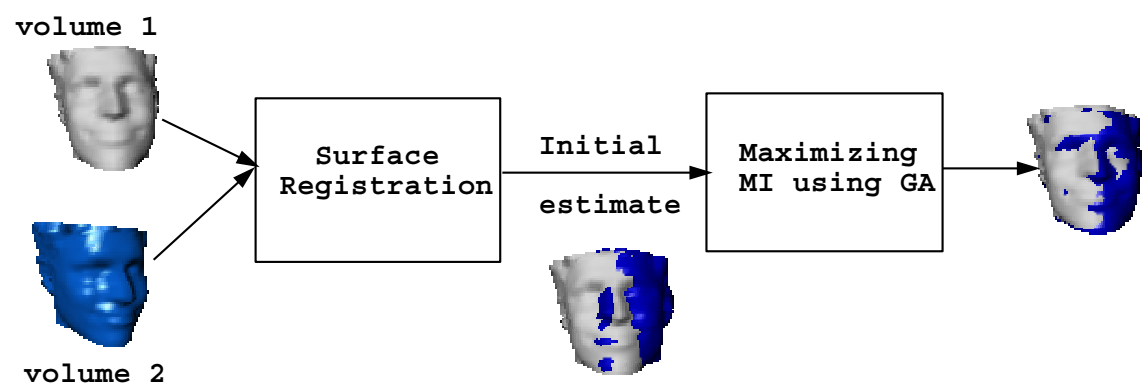

Fig. 1. The block diagram of the proposed system. Two different volumes are fed into the system. Surface registration provides an estimate transformation between them. This result is further enhanced by maximizing the mutual information. A Genetic Algorithms technique is used in the search process.

\section{Surface Registration Technique}

In order for any surface registration algorithm to perform accurately and efficiently, an appropriate representation scheme for the surface is needed. Most of the surface representation schemes found in literature have adopted some form of shape parameterization especially for the purpose of object recognition. However, free-form surfaces, in general, may not have simple volumetric shapes that can be expressed in terms of parametric primitives. This paper presents a new concept, introduced in [1,2], for 3-D free-form surface registration and object recognition using a new surface representation scheme. This representation scheme captures the 3-D curvature information of any free-form surface and encodes it into a 2-D image corresponding to a certain point on the surface. This image is unique for this point and is independent from the object translation or orientation in space. The process starts by identifying special points on the surface. These points are called Important points due to the information they carry. Then an image capturing the surface curvature information seen from each important point, is formed. We call this image the Surface Point Signature (SPS) because it is almost unique for each point on the surface. Surface registration is then performed by matching SPS images of different surfaces and hence finding corresponding points in each surface. For rigid registration, three point correspondences are sufficient to estimate the transformation parameters.

Definition 1. A point $P$ on a surface/curve $S$, is called important point, $P_{I}$, if and only if the absolute value of the curvature at this point is larger than a certain positive value (a threshold).

$$
A=\left\{P_{I}\right\}=\{P \in S|| C u r v(P) \mid>\epsilon, \epsilon>0\}
$$

As the important points are landmarks, one may expect that they are stable for the same object. However, due to scanning noise, their number and locations 

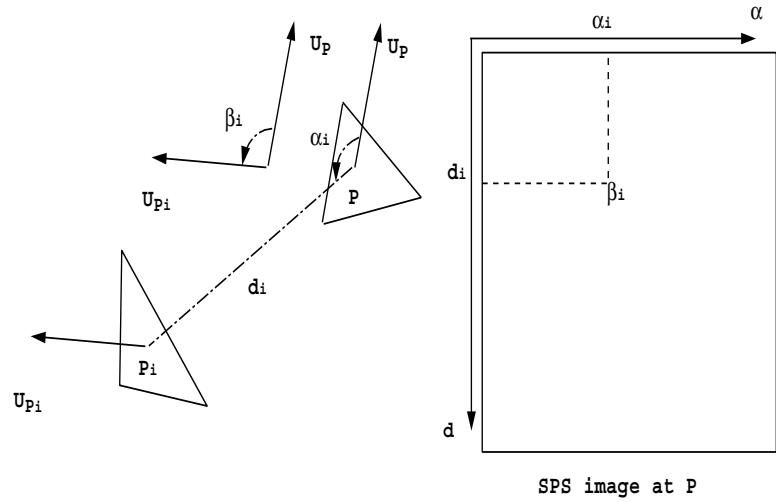

(a)

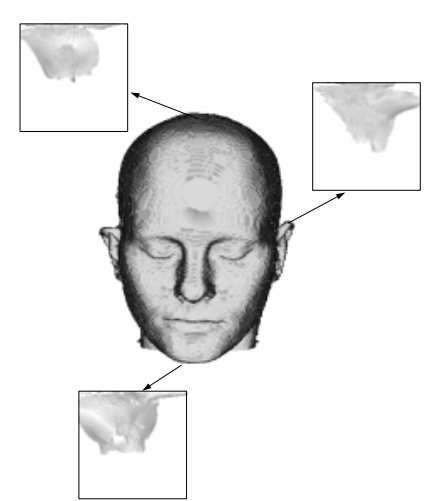

(b)

Fig. 2. (a) For each important point on the surface an SPS image is obtained. This image encodes the angle difference between the normal at the point in focus and the normal at each other point on the surface. (b) Examples of SPS images taken at different important points locations. Notice how the image provides features the curvature information. The dark intensity in the image represents a high curvature seen from the point while the light intensity represents a low curvature. Also notice how different the image corresponding to a location is from images of other locations.

may vary. By adjusting the curvature threshold, a common subset can be found. Otherwise, the object has either suffered from non-rigid transformations or its visible surface has no landmarks.

Our approach for fast registration and recognition is to establish a "surface signature" for each of the obtained 3-D important points rather than just depending on the 3-D coordinates of the point.

The signature, computed at each important point, encodes the surface curvature seen from this point, thus giving it discriminating power. As shown in Figure 2 (a), for each important point $P \in A$ defined by its 3 -D coordinates and the normal $U_{P}$ at the patch where $P$ is the center of gravity, each other point $P_{i}$ on the surface can be related to $P$ by two parameters: 1$)$ The distance $d_{i}=\left\|P-P_{i}\right\|$ and 2) the angle $\alpha_{i}=\cos ^{-1}\left(\frac{U_{P} \cdot\left(P-P_{i}\right)}{\left\|P-P_{i}\right\|}\right)$. This is a polar implementation of the SPS image, and it can be easily converted into Cartesian form. Also we can notice that there is a missing degree of freedom in this representation which is the cylindrical angular parameter. This parameter depends on the surface orientation which defies the purpose of having an orientation-independent representation scheme. The SPS generation is scale-dependent. However, this has a little effect on its use in multi-modal registration since the result is refined in the volume matching stage and also since there is no or only a small scale dif- 
ference between the volumes in study. At each location in the image, we encode the angle $\beta_{i}=\cos ^{-1}\left(U_{P} \cdot U_{P_{i}}\right)$. This represents the change in the normal at the surface point $P_{i}$ relative to the normal at $P$. Due to the fact that we are ignoring the cylindrical angular degree, the same pixel in the SPS image can represent more than one $3-\mathrm{D}$ point on the surface. This usually occurs when we have a surface of revolution around the axis represented by the normal at the point $P$. As all these points have the same $d_{i}$ and $\alpha_{i}$ and lie on the circle that has a radius $d_{i} \cos \left(\alpha_{i}\right)$ and is distant by $d_{i} \sin \left(\alpha_{i}\right)$ from the point $P$ along the axis $U_{P}$, we take the average of their angles $\beta_{i}$ and encode it in the SPS corresponding pixel location.

Figure 2 (b) shows some SPS images taken at different important points on the skin model. We can see clearly how the images encode the curvature information of the object surface and each uniquely defines the location of the point on the surface.

The next step in the registration process is to match corresponding SPS images of two surfaces. The ultimate goal of the matching process is to find at least a three points correspondence in order to calculate the transformation parameters. The benefit of using the SPS images to find the correspondence is that we can now use image processing tools in the matching, hence reducing the time taken to find accurate transformation. One such tool is Template Matching in which a value defines how well a portion of an image matched a template.

The end result of the matching process is a list of groups of likely three point correspondences that satisfies the geometric consistency constraint. The list is sorted such that correspondences that are far apart are at the top of the list. A rigid transformation is calculated for each group of correspondences and a verification stage [3] is performed to obtain the best group. Detailed discussion concerning the SPS sensitivity and robustness can be found in [1].

\section{Volume Registration Technique}

Extracting skin from MR dataset using an automatic segmentation technique may produce an inaccurate $3-\mathrm{D}$ skin model. In such a case, we start with the results (estimation of transformation parameters) of our surface registration as initial parameters for a volume registration as shown in Figure 1. We used the well-known maximization of Mutual Information (MI) as a matching criterion based on Genetic Algorithms (GA) as a search engine. The search is performed around the initial transformation parameters estimated by the surface registration technique. As a result, we need a fewer number of iterations than with volume registration that starts with random transformation parameters. MI is a basic concept from information theory, measuring the statistical dependence between two random variables or the amount of information that one variable contains about the other. The MI registration criterion used here states that the MI of corresponding voxel pairs is maximal if the two volumes are geometrically aligned [4]. No assumptions are made regarding the nature of the relation between the image intensities in either modality. 
We refer to the two medical volumes to be registered as the reference volume $R$ and the floating volume $F$. A voxel of the reference volume is denoted $R(x)$, where $x$ is the coordinates vector of the voxel. A voxel of the floating volume is denoted similarly as $F(x)$. Given that $T$ is a transformation matrix from the coordinate space of the reference volume to the floating volume, $F(T(x))$ is the floating volume voxel associated with the reference volume voxel $R(x)$. Note that in order to simplify the MI equation we will use $T$ to denote both the transformation and its parameterization.

We seek an estimate of the transformation matrix that registers the reference volume $R$ and floating volume $F$ by maximizing their mutual information. The vector $x$ is treated as a random variable over coordinate locations in the reference volume. Mutual information is defined in terms of entropy in the following way [5]:

$$
I(R(x), F(T(x))) \equiv h(R(x))+h(F(T(x)))-h(R(x), F(T(x))) .
$$

where $h(R(x))$ and $h(F(T(x)))$ are the entropy of $R$ and $F$, respectively. $h(R(x), F(T(x)))$ is the joint entropy. Entropy can be interpreted as a measure of uncertainty, variability, or complexity. The mutual information defined in equation (2) has three components. The first term on the right is the entropy in the reference volume, and is not a function of $T$. The second term is the entropy of the part of the floating volume into which the reference volume projects. It encourages transformations that project $R$ into complex parts of $F$. The third term, the (negative) joint entropy of $R$ and $F$, contributes when $R$ and $F$ are functionally related. Maximizing MI tends to find as much of the complexity that is in the separate volumes (maximizing the first two terms) as possible so that at the same time they explain each other well (minimizing the third term) $[4,5]$.

GA are adaptive, domain independent search procedures derived from the principles of natural population genetics. GA are briefly characterized by three main concepts: a Darwinian notion of fitness or strength which determines an individual's likelihood of affecting future generations through reproduction; a reproduction operation which selects individuals for recombination to their fitness or strength; and a recombination operation which creates new offspring based on the genetic structure of their parents. GA work with a coding of a parameter set, not the parameters themselves, and search from a population of points, not a single point [6]. Also, GA use payoff (objective function) information, not derivatives or other auxiliary knowledge, and employ probabilistic transition rules instead of deterministic ones. These four differences contribute to genetic algorithms' robustness and resulting advantage over other more commonly used search techniques. Our results demonstrate that MI, combined with a GA search, is a robust approach for multi-modal volume registration [7].

\section{Results}

We evaluated our results with experimental as well as with real datasets. For the experimental test, we implemented a tool which applies a certain rotation 

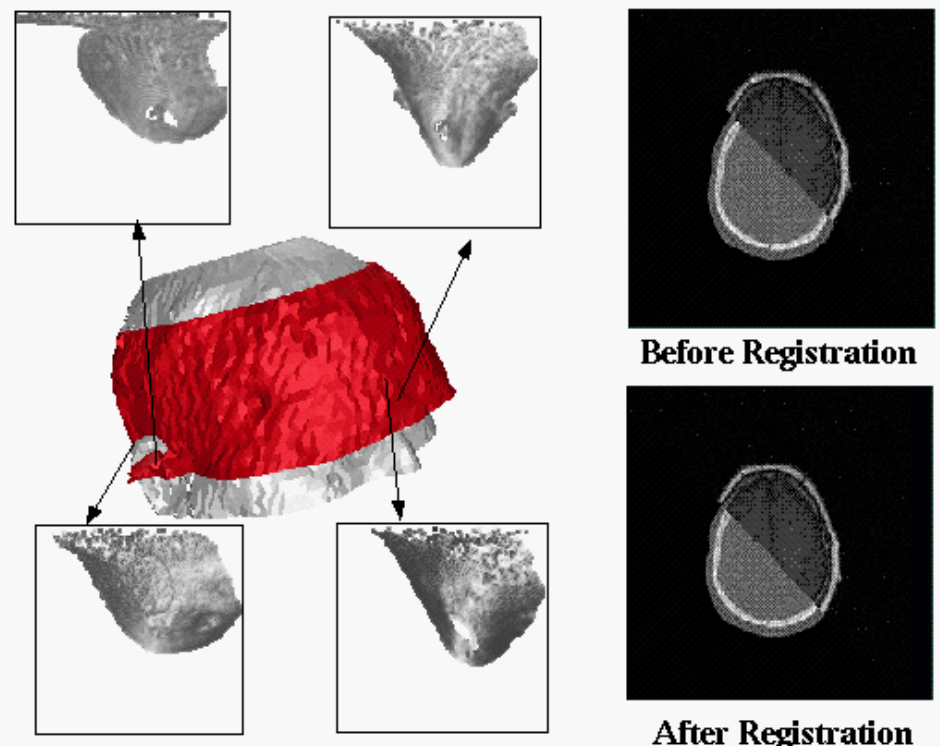

After Registration

Fig. 3. Two different SPS samples. The upper two images are from the MR dataset; while the lower two are from the CT dataset. This figure shows similarity of the SPS images of each matched pair, although they are produced from two different datasets (CT and $M R$ ) with a different 3-D volume size (number of slices).

and translation on a $3-\mathrm{D}$ medical volume. We start with a $3-\mathrm{D}$ volume $V$. Using our transformation tool, we produce a transformed volume $V^{\prime}$ with a known transformation matrix. Next, we apply our registration technique on the two volumes $V$ and $V^{\prime}$ to obtain an estimate of the transformation matrix. Registration accuracy is measured by comparing the estimated results with the actual transformation parameters. We also evaluated the case of registering two different modalities, such as CT and MR, from the same patient (real datasets). To validate the results of the new technique, a ground truth was obtained by an exhaustive search performed on a 24-processors ONYX R10000 SGI machine. Registration accuracy is measured visually and/or by comparing the estimated results with the transformation parameters determined by the exhaustive search. Figures 3 and 4 show a sample of our results of $\mathrm{CT} / \mathrm{CT}$ and $\mathrm{CT} / \mathrm{MR}$ alignment. The SPS images shown in Figures 3 and 4 clearly show that each SPS image is unique at a certain point and is independent from $3-\mathrm{D}$ volume translation or orientation in the space. A mathematical proof is presented in [1].

In comparing these results with conventional MI multi-modal registration techniques [7], it is found that using surface registration a priori to maximize the MI reduces the registration time while resulting in the same accuracy. The results 

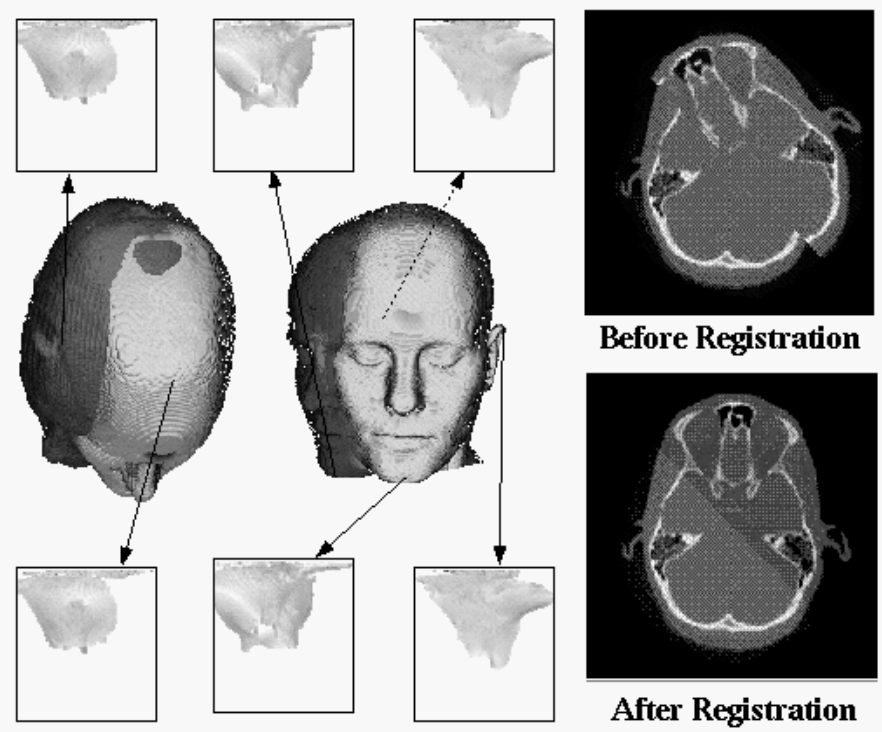

Fig. 4. Three different matched pairs of SPS images produced from a CT volume. This figure shows that the SPS images are independent from the 3-D volume translation or orientation in the space.

are summarized in Table 1 and compared with the results of using a single-stage multi-modal volume registration technique presented in [7]. The new surface registration is also faster than existing approaches. The GA also contributes in the fast conversion. The time required for registration is approximately two to three minutes for high resolution volumes.

Table 1. Comparison between approximate times for single and dual stage volume registration

\begin{tabular}{|c|c|c|c|c|c|c|}
\hline \multirow{3}{*}{ Case } & \multirow{3}{*}{$\begin{array}{l}\text { No of } \\
\text { slices }\end{array}$} & \multicolumn{4}{|c|}{ Dual-Stage } & \multirow{3}{*}{\begin{tabular}{|c|} 
Single-Stage \\
MI-GA \\
$(\mathrm{sec})$
\end{tabular}} \\
\hline & & \multicolumn{2}{|c|}{ Surface Matching } & \multirow{2}{*}{$\begin{array}{c}\text { Volume } \\
\text { Matching }\end{array}$} & \multirow{2}{*}{$\begin{array}{c}\text { Total time } \\
(\mathrm{sec})\end{array}$} & \\
\hline & & SPS Generation & SPS Matching & & & \\
\hline $\mathrm{CT} / \mathrm{CT}$ & 236 & $20 \mathrm{sec}$ & $30 \mathrm{sec}$ & $100 \mathrm{sec}$ & 150 & 300 \\
\hline $\mathrm{CT} / \mathrm{MR}$ & $33 / 19$ & $8 \mathrm{sec}$ & $20 \mathrm{sec}$ & $80 \mathrm{sec}$ & 108 & 250 \\
\hline
\end{tabular}




\section{Conclusion and Future Work}

Multi-modal medical volume registration is an important capability for finding the correct geometrical transformation that brings one volume in precise spatial correspondence with another. Multi-modal volume registration allows us to benefit from the complementary information in medical volumes from different modalities. Implementing a fast and accurate registration technique is one of the preprocessing stages during neursurgical procedures. The results demonstrate that our registration technique provides fast, accurate, robust and completely automatic registration of multimodality medical volumes. Future work is to incorporate volume deformation in order to register preoperative CT or MR volumes quickly and accurately with intraoperative MR volumes produced by an open-magnet system.

\section{Acknowledgments}

This research work has been partially supported by a grant from the Alliant Health System, Louisville, Kentucky, USA. The authors acknowledge Dr. J. Fitzpatrick and Dr. Jay West from Vanderbilt University for providing access to their multi-modal volume datasets from the Retrospective Registration Evaluation NIH Project (project number R01 NS33926-01).

\section{References}

1. S. M. Yamany and A. A. Farag, "Free form surface maching using surface signatures," International Conference on Computer Vision and Pattern Recognition ICCV'99, Corfu, Greece, Sept. 20 - 25, 1999. 673, 675, 677

2. S. M. Yamany, A. El-Bialy, and A. A. Farag, "Free form object recognition and registration using surface signatures," IEEE International Conference on Image Processing - ICIP'99, Japan, Oct. 25 - 28, 1999. 673

3. S. M. Yamany, M. N. Ahmed, and A. A. Farag, "A new genetic-based technique for matching 3d curves and surfaces," Pattern Recognition (to appear), 1999. 675

4. F. Maes, A. Collignon, D. Vandermeulen, G. Marchal, and P. Suetens, "Multimodality image registration by maximization of mutual information," IEEE Transaction on Medical Imaging 16(2), pp. 187-198, 1997. 675, 676

5. W. M. Wells, P. Viola, H. Atsumi, S. Nakajima, and R. Kikinis, "Multi-model volume registration by maximization of mutual information," Medical Image Analysis, Oxford University Press 1(1), pp. 35-51, 1996. 676

6. D. E. Goldberg, Genetic Algorithms in Search, Optimization and Machine Learning, Addison-Welsey, 1989. 676

7. A. Eldeib, A. A. Farag, and T. Moriarty, "A fast genetic search algorithm for accurate multi-modal volume registration by maximization of mutual information," Computer Assisted Radiology and Surgery - CARS'99, 13th International Congress and Exhibition, Paris, France, June 23 - 26 , 1999. 676, 677, 678 